\section{MECHANICAL THROMBECTOMY IN ACUTE ISCHEMIC STROKE PATIENTS WITH LOW ALBERTA STROKE PROGRAM EARLY COMPUTED TOMOGRAPHY SCORES}

${ }^{1} \mathrm{O}$ Zaidat*, ${ }^{2} \mathrm{D}$ Liebeskind, ${ }^{3} \mathrm{~A}$ Jadhav, ${ }^{4} \mathrm{~S}$ Ortega-Gutierrez, ${ }^{2} \mathrm{~V}$ Szeder, ${ }^{5} \mathrm{D}$ Haussen, ${ }^{6} \mathrm{D}$ Yavagal, ${ }^{7} \mathrm{M}$ Froehler, ${ }^{2} \mathrm{R}$ Jahan, ${ }^{8} \mathrm{~T}$ Yao, ${ }^{9} \mathrm{~N}$ Mueller-Kronast. ${ }^{1}$ Bon Secours Mercy Health St. Vincent Medical Center, Toledo, OH; ${ }^{2}$ UCLA, Los Angeles, CA; ${ }^{3}$ UPMC Presbyterian Hospital, Pittsburgh, PA; ${ }^{4}$ University of lowa, lowa City, IA; ${ }^{5}$ Emory University School of Medicine, Atlanta, GA; ${ }^{6}$ University of Miami, Miami, FL; ${ }^{7}$ Vanderbilt University Medical Center, Nashville, $T N_{i}^{8}$ Norton Neuroscience Institute, Louisville, $K Y ;{ }^{9}$ Delray Medical Center, Delray Beach, FL

\subsection{6/neurintsurg-2019-SNIS.228}

Background and purpose Limited data exists on the benefit of mechanical thrombectomy (MT) in acute ischemic stroke patients presenting with low ASPECTS (Alberta Stroke Program Early Computed Tomography (CT) Score). The aim of this substudy was investigate the outcome of low ASPECTS (0-5) patients undergoing mechanical thrombectomy in the Systematic Evaluation of Patients Treated With Neurothrombectomy Devices for Acute Ischemic Stroke (STRATIS) Registry.

Methods Data from the STRATIS Registry, a prospective, multicenter, non-randomized, observational study of AIS LVO patients treated with the Solitaire stent-retriever as the first choice therapy within 8 hours from symptoms onset, was used to identify patients with baseline ASPECTS 0-5. CT ASPECTS was adjudicated by a core lab blinded to clinical outcomes.

Results A total of $57 / 763$ (7.5\%) patients had a baseline ASPECTS $0-5$, of which 10 were ASPECTS $0-3$ and 47 ASPECTS 4-5. Mean baseline NIHSS was 19.9 \pm 5.1 . The majority of patients presented with ICA (42.1\%) and M1 (47.4\%) occlusions. IV-rtPA was administered in 68.4\%. Mean onset to arterial puncture was $276 \pm 102.9$ minutes and puncture to reperfusion time was $45.3 \pm 25.3$ minutes. The majority of patients $(85.5 \%)$ achieved substantial reperfusion (mTICI $\geq 2 b)$. Ninety-day outcome was reported in 52/57 $(91.2 \%)$. The rate of good functional outcome (mRS $\leq 2)$ was $28.8 \%$ (versus $59.7 \%$ in ASPECTS $6-10$ group, $\mathrm{p}<0.001$ ), which is higher than the $14.1 \%$ reported in the control arm $0-5$ in the HERMES pooled analysis. Symptomatic intracranial hemorrhage and mortality rates were $7.0 \%$ and $30.8 \%$, respectively. When further dichotomizing the group to ASPECTS $0-3$ and 4-5 to determine the cut-off for MT futility, the rate of good outcome was $10 \%$ and $33.3 \%$, respectively. In investigating the interaction between age and ASPECTS 0-5, low ASPECTS patients older than 75 had a lower rate of good clinical outcome than those 65-75 and less than 65 (0\%, 18.2\%, 44.8\%).

Conclusion In the STRATIS Registry, low ASPECTS $0-5$ is associated with lower functional outcomes in patients undergoing mechanical thrombectomy. Clinical outcome in low ASPECTS may be age dependent. Prospective studies are needed to understand the benefit of MT in this patient population.

Disclosures O. Zaidat: None. D. Liebeskind: None. A. Jadhav: None. S. Ortega-Gutierrez: None. V. Szeder: None. D. Haussen: None. D. Yavagal: None. M. Froehler: None. R. Jahan: None. T. Yao: None. N. Mueller-Kronast: None.

\section{6} ROBOTIC-ASSISTED NEUROVASCULAR INTERVENTION

${ }^{1} \mathrm{G}$ Britz ${ }^{*}{ }^{2} \mathrm{~J}$ Tomas, ${ }^{1} \mathrm{~A}$ Lumsden. ${ }^{1}$ Houston Methodist Hospital, Houston, $T X_{i}{ }^{2}$ Corindus Inc., Waltham, MA, USA

\subsection{6/neurintsurg-2019-SNIS.229}

Background Despite advances in robotic-assisted technology for cardiac and peripheral vascular interventions, a roboticassisted platform for neurovascular intervention is not yet available.

Objective The goal of this preclinical study was to evaluate the feasibility of the CorPath GRX robotic-assisted platform (Corindus Inc., Waltham, MA, USA) for neurovascular interventions including stroke.

Methods The robotic system was tested for its ability to accurately navigate a variety of common neurovascular devices in an in vitro flow model and in a live, anesthetized pig, under conditions and following procedures appropriate for clinical intervention. An access catheter was introduced manually at the equivalent of the common carotid artery in both models. Endovascular wires and catheters were navigated through the external and internal carotid artery and posterior cerebral vasculature under robotic assistance, using 0.014 in guidewires, 2.4F/1.7F microcatheters, bare-metal stents, and embolic coils. Results All procedures in both the flow and porcine models, including navigation, wiring, and deployment of the stent, and coils, were performed successfully with no technical complications. There was no evidence of extravasation, dissection, thrombosis, or other vascular injury when angiography was compared before and after the live-animal procedure.

Conclusion This is the first study to demonstrate that use of a robotic-assisted platform is feasible for intracranial neurovascular intervention. The robotic system was successful at navigating and deploying the small-gauge devices specific to neurovascular procedures. Given the potential benefits of robotic-assisted surgery for the patient and the surgeon, further investigation is warranted for this indication.

Disclosures G. Britz: 1; C; Houston Methodist. J. Tomas: None. A. Lumsden: 4; C; Corindus Inc.

\section{MECHANICAL THROMBECTOMY FOR ACUTE BASILAR ARTERY OCCLUSION}

${ }^{1} \mathrm{H}$ Liu* ${ }^{*} \mathrm{C}$ Lee, ${ }^{2} \mathrm{Y}$ Lin, ${ }^{1} \mathrm{C}$ Wan. ${ }^{1} \mathrm{Fu}$ Jen Catholic University Hospital, New Taipei City, Taiwan; ${ }^{2}$ National Taiwan University Hospital, Taipei, Taiwan

\subsection{6/neurintsurg-2019-SNIS.230}

Purpose The present our experience in mechanical thrombectomy for acute basilar artery occlusion.

Method and material By reviewing our databank, in the last 300 patients of acute cerebral ischemic stroke of large vessel occlusion, we found 29 patients were due to basilar artery occlusion (BAO). The inclusion criteria were; $>18$ years old, no evidence of hemorrhage on CT, BAO found on CTA, bridging therapy after IV-tPA in eligible patients, posterior circulation ASPECTS $>6$. We recorded the initial NIHSS, time from onset to needle, method of recanalization, TICI, time of 
recanalization, 24- hour follow up imaging, and mRS at 90 days.

Result In these 29 patients, they were 14 were males, 68 years old (42-81 years old). The initial NIHSS at emergency department was 27 (standard deviation $(\mathrm{SD})=7.3$ ); 8 of them received IV-tPA, the average time from onset to needle was 417 minutes ( $\mathrm{SD}=38.9)$; 93.1\% (27/29) using aspiration as first method of recanalization; TICI $=3$ or $2 \mathrm{~b}$ in $86.2 \%(25 /$ $29)$; 41.4\% (12/29) associated with basilar artery stenosis; on the 24-hour follow up imaging, parenchymal hemorrhage was found in 3 and hemorrhagic transformation in 3; the mRS $(0-2)$ at 90 days was $55.1 \%(16 / 29)$, and the mortality rate was $10.3 \%(3 / 29)$.

Conclusion Acute basilar artery occlusion is a complex and severe disease, endovascular thrombectomy can have a high rate of recanalization but the clinical outcome on 90 days still worse than the one in anterior circulation. The treatment will become more challenge in patient associated with underlying intracranial atherosclerotic stenosis.

Disclosures H. Liu: 3; C; Stryker, GE. C. Lee: None. Y. Lin: None. C. Wan: None.

\section{INTRA-ARTERIAL THROMBOLYSIS AS RESCUE THERAPY IN MCA OCCLUSIONS: SUBANALYSIS FROM THE STRATIS REGISTRY}

${ }^{1} \mathrm{~A}$ Castonguay*, 'S Zaidi, 'M Jumaa, ${ }^{2} \mathrm{O}$ Zaidat, ${ }^{3} \mathrm{D}$ Liebeskind, ${ }^{1} \mathrm{H}$ Salahuddin, ${ }^{4} \mathrm{~N}$ MuellerKronast. 'University of Toledo, Toledo, OH; ${ }^{2}$ Mercy St. Vincent, Toledo, OH; ${ }^{3} \mathrm{UCLA}$, Los Angeles, CA; ${ }^{4}$ Delray Medical Center, Delray Beach, FL

\subsection{6/neurintsurg-2019-SNIS.231}

Background and purpose Recent data suggests that intra-arterial (IA) thrombolytics may be a safe rescue therapy (RT) for acute ischemic stroke (AIS) patients after failed mechanical thrombectomy (MT); however, its safety and efficacy in MCA occlusions remains unclear. Here, we investigate the use of IA tissue plasminogen activator (IA-rtPA) as RT in MCA occlusions in the Systematic Evaluation of Patients Treated With Neurothrombectomy Devices for Acute Ischemic Stroke (STRATIS) Registry.

Methods The STRATIS Registry was a prospective, multicenter, non-randomized, observational study of AIS LVO patients treated with the Solitaire stent-retriever as the first choice therapy within 8 hours from symptoms onset. Clinical and angiographic outcomes were compared in patients with MCA occlusions treated with and without IA-rtPA.

Results A total of $680 / 938$ (72.5\%) patients with IA-tPA use harbored MCA occlusions, of which 585 (86.0\%) and 95 $(14.0 \%)$ were in the no IA-rtPA and IA-rtPA groups, respectively. Baseline demographics were well-balanced between the cohorts. The majority of MCA occlusions were located in the M1 segment, with $76.1 \%$ in the no IA-rtPA group and $74.7 \%$ in the IA-rtPA group $(\mathrm{p}=0.80)$. IV-rtPA was administered in $64.4 \%$ and $71.6 \%$ of the no IA-rtPA and IA-rtPA patients $(p=0.20)$. Median IA-rtPA dose was 4mg (IQR 2$10)$ in the IA-rtPA cohort. Onset to puncture time was less than 6 hours in $88.6 \%(597 / 674)$ of patients. Mean onset to arterial puncture time was shorter in the IA-rtPA group (196.7 \pm 109.9 versus 228.2 \pm 97.9 minutes, $p=0.004)$; however, mean puncture to procedure end time was longer in the IA-rtPA group than the no IA-rtPA group $(74.5 \pm 41.5$ versus $59.5 \pm 33.6$ minutes). Mean number of passes $(2.0 \pm 1.2$ versus $1.6 \pm 1.0, \mathrm{p}=0.005)$ and rate of distal embolization
(53.4\% versus $72.1 \%, p=0.001)$ were significantly higher in the IA-rtPA group. The rate of substantial reperfusion $(\mathrm{mTICI} \geq 2 \mathrm{~b})$ was similar in the cohorts $(89.9 \%$ versus $86.0 \%, \mathrm{p}=0.28)$. Rates of symptomatic intracranial hemorrhage $(\mathrm{sICH})(1.7 \%$ versus $2.2 \%, \mathrm{p}=0.68)$, good functional outcome (mRS $\geq 2,60.5 \%$ versus $58.0 \%, \mathrm{p}=0.85$ ), and mortality $(13.9 \%$ versus $13.6 \%, p=0.96)$ at 90 days did not differ between the two groups.

Conclusion Use of IA-rtPA as rescue therapy after failed mechanical thrombectomy in MCA occlusions was not associated with an increased risk of sICH or mortality in the STRATIS Registry. More studies are needed to understand the safety and efficacy of IA thrombolysis as rescue therapy in this patient population.

Disclosures A. Castonguay: None. S. Zaidi: None. M. Jumaa: None. O. Zaidat: None. D. Liebeskind: None. H. Salahuddin: None. N. Mueller-Kronast: None.

\section{FACTORS ASSOCIATED WITH THE DECISION-MAKING ON ENDOVASCULAR THROMBECTOMY FOR FHE MANAGEMENT OF ACUTE ISCHEMIC STROKE}

${ }^{1} \mathrm{M}$ Goyal ${ }^{*},{ }^{2} \mathrm{~B}$ Menon, ${ }^{3} \mathrm{~N}$ Kashani, ${ }^{3} \mathrm{~A}$ Wilson, ${ }^{4} \mathrm{~S}$ Yoshimura, ${ }^{5} \mathrm{~B}$ Campbell, ${ }^{6} \mathrm{~B}$ Baxter, ${ }^{7} \mathrm{~A}$ Rabinstein, ${ }^{8} \mathrm{~F}$ Turjman, ${ }^{9} \mathrm{U}$ Fischer, ${ }^{10} \mathrm{~J}$ Ospel, ${ }^{11} \mathrm{~W}$ Kunz, ${ }^{12} \mathrm{P}$ Mitchell, ${ }^{13} \mathrm{P}$ Sylaia, ${ }^{14} \mathrm{M}$ Cherian, ${ }^{15} \mathrm{~B}$ Kim, ${ }^{16} \mathrm{~J} \mathrm{Heo},{ }^{17} \mathrm{~A}$ Podlasek, ${ }^{3} \mathrm{M}$ Almekhlafi, ${ }^{3} \mathrm{M}$ Foss, ${ }^{18} \mathrm{~A}$ Demchuk, ${ }^{18} \mathrm{M}$ Hill, ${ }^{19} \mathrm{G}$ Saposnik. ${ }^{1}$ Radiology, Clinical Neurosciences, University of Calgary, Calgary, $A B$, Canada; ${ }^{2}$ Clinical Neurosciences, University of Calgary, Calgary, $A B$, Canada; ${ }^{3}$ Radiology, University of Calgary, Calgary, $A B$, Canada; ${ }^{4}$ Neurosurgery, Hyogo College of Medicine, Hyogo, Japan; ${ }^{5}$ Neurology, Royal Melbourne Hospital, University of Melbourne, Melbourne, Australia; ${ }^{6}$ Radiology, Erlanger Hospital Chattanooga, Chattanooga, TN; ${ }^{7}$ Radiology, Mayo Clinic Rochester, Rochester, MN; ${ }^{8}$ Radiology, Centre Hospitalier Universitaire de Lyon, Lyon, France; ${ }^{9}$ Radiology, University Hospital Bern (Inselspital), Bern, Switzerland; ${ }^{10}$ Radiology, University Hospital Basel, Basel, Switzerland; ${ }^{11}$ Radiology, University Hospital Munich, Munich, Germany; ${ }^{12}$ Radiology, Royal Melbourne Hospital, University of Melbourne, Melbourne, Australia; ${ }^{13}$ Radiology, Sree Chitra Tirunal Institute for Medical Sciences and Technology, Thiruvananthapuram, India; ${ }^{14}$ Radiology, Kovai Medical center, Tamil Nadu, India; ${ }^{15}$ Radiology, St. Mary's Hospital Seoul, Seoul, Korea, Republic of; ${ }^{16}$ Neurology, Yonsei University, Seoul, Seoul, Korea, Republic of; ${ }^{17}$ Radiology, Southend University Hospital NHS Foundation Trust, Westcliff-on-Sea, UK; ${ }^{18}$ Neurology, University of Calgary, Calgary, AB, Canada; ${ }^{19}$ Neurology, St. Michael's hospital, Toronto, ON, Canada

\subsection{6/neurintsurg-2019-SNIS.232}

Background and Purpose Little is known about the real-life factors that clinicians use in selection of patients that would receive endovascular treatment (EVT) in the real world. We sought to determine patient, practitioner, and health system factors associated with therapeutic decisions around endovascular treatment.

Methods We conducted a multinational cross-sectional webbased study comprising of 607 clinicians and interventionalists from 38 countries who are directly involved acute stroke care. Participants were randomly allocated to 10 from a pool of 22 acute stroke case scenarios and stated how they would treat the patient A) under current local resources, and B) under assumed ideal conditions (i.e. without monetary or infrastructural constraints). Descriptive statistics and logistic regression was performed to determine multilevel factors associated with EVT, adherence to current EVT guidelines, and practice gaps between current local resources and assumed ideal conditions. Results Of the 1330 invited participants, 607 (45.6\%) participants completed the study $(53.7 \%$ neurologists, $28.5 \%$ neurointerventional radiologists, $17.8 \%$ other clinicians). NIHSS (34.9\%), level of evidence (30.2\%), ASPECTS or ischemic core volume (22.4\%), patient's age (21.6\%), and clinicians' 